\title{
Genome-wide meta-analysis reveals novel susceptibility loci for thyrotoxic periodic paralysis
}

\author{
Gloria Hoi-Yee Lii ${ }^{1,2}$, Ching-Lung Cheung ${ }^{\mathbb{1}}$, Shuang-Xia Zhao ${ }^{3}$, Huai-dong Song ${ }^{3}$ and Annie Wai-Chee Kung ${ }^{4}$
}

'Department of Pharmacology and Pharmacy, Li Ka Shing Faculty of Medicine, The University of Hong Kong, Hong Kong, ${ }^{2}$ Department of Health Technology and Informatics, The Hong Kong Polytechnic University, Hung Hom, Hong Kong, ${ }^{3}$ Department of Molecular Diagnostics, The Core Laboratory in Medical Center of Clinical Research, Department of Endocrinology, Shanghai Ninth People's Hospital, State Key Laboratory of Medical Genomics, Shanghai Jiaotong University School of Medicine, Shanghai, China, and ${ }^{4}$ Department of Medicine, Li Ka Shing Faculty of Medicine, The University of Hong Kong, Hong Kong

Correspondence should be addressed to C-L Cheung Email

lung1212@hku.hk

\begin{abstract}
Objective: Thyrotoxic periodic paralysis (TPP) is a rare and potentially fatal complication of hyperthyroidism. By meta-analysis of genome-wide association studies, we aim to discover novel susceptibility loci and understand the pathogenesis of TPP.

Methods: This meta-analysis comprised 319 TPP cases and 3516 healthy controls from three independent cohorts (two from Hong Kong; one from Shanghai). Genetic variants in each cohort were separately genotyped, imputed and analyzed for association with TPP. Fixed-effect meta-analysis was performed to combine the data. Using the three independent genome-wide significant variants, a weighted genetic risk score (GRS) was developed.

Results: Of 7077246 variants tested for association with TPP, 260 variants reached genome-wide significance and were represented by independent variants from four distinct genomic loci, but a risk locus for Graves' disease at 6p21.33-p21.22 was excluded from subsequent analyses. Two novel loci near TRIM2 (4q31.3; rs6827197: OR = 4.075; $\left.P=3.46 \times 10^{-9}\right)$ and $A C 140912.1\left(16 q 22.3 ; \mathrm{rs} 6420387: \mathrm{OR}=1.861 ; P=2.66 \times 10^{-8}\right)$ were identified. Together with previously reported $K C N / 2\left(17 q 24.3 ; r s 312743: O R=2.564 ; P=1.15 \times 10^{-21}\right)$, the three susceptibility variants explained $4.36 \%$ of the genetic liability. Expression quantitative trait loci analyses showed the variants altered expression of TRIM2 in nerve and KCNJ2 in skeletal muscle. The weighted GRS had an area under curve of 0.827 and 0.682 in the derivation and validation cohorts in Hong Kong.

Conclusions: We identified two novel TPP risk loci near TRIM2 and AC140912.1. While rare mutations in TRIM2 and KCNJ2 were implicated in monogenic disorders characterized by muscle paralysis, our study suggested common variants near these genes might dysregulate gene expression and lead to milder phenotypes.
\end{abstract}

\section{Introduction}

Thyrotoxic periodic paralysis (TPP) is a rare and potentially fatal complication of hyperthyroidism characterized by recurrent hypokalemia, episodic muscle weakness and paralysis (1). In serious attacks, life-threatening cardiopulmonary complications, such as ventricular arrhythmia, total paralysis of respiratory and bulbar (c) 2020 European Society of Endocrinology Printed in Great Britain muscles, may also occur (1). While it mainly affects males in Asians with a prevalence of $13 \%$ among male patients with thyrotoxicosis in Chinese, the overall incidence of TPP in Chinese is $1.8 \%$ (1). Despite the predominance in male, the ratio of male to female with TPP varied from 17:1 to $76: 1$ (2). Following migration and increased awareness

Published by Bioscientifica Ltd. 
of the condition, more TPP cases have been observed in various populations in Western countries (3).

As episodic muscle weakness and paralysis in TPP are similar to known channelopathies like hypokalemia periodic paralysis and Andersen-Tawil syndrome (ATS), TPP was recognized to be a channelopathy. We previously identified mutations in an unreported gene, potassium inwardly rectifying channel subfamily J member 18 (KCNJ18) encoding the inwardly rectifying potassium (KIR) channel KIR2.6, were present in approximately one-third of TPP patients (4). On the other hand, using genome-wide association study (GWAS), we identified another member of KIR channels, KCNJ2, as a susceptibility gene of TPP, and significant association with $K C N J 2$ was consistently observed in other GWAS of $\operatorname{TPP}(5,6,7)$. In the latest single-cohort GWAS, a novel genome-wide significant TPP locus unrelated to ion channel was revealed in dachsous cadherin-related 2 (DCHS2) (7). In addition to the loci near KCNJ2 and a suggestive locus near C11orf67, the three susceptibility loci contributed to $3.1 \%$ heritability of the disease (7) but they could not fully explain the genetic liability of TPP.

In the present study, we aimed to dissect the genetics of TPP by firstly performing an additional GWAS in a southern Chinese cohort in Hong Kong with 92 TPP cases and 2077 controls, then conducted a meta-analysis with two published GWAS $(7,8)$, totaling 319 TPP cases and 3516 healthy controls. To enhance early identification of the disease, the TPP-associated genetic variants derived from the meta-analysis were employed to develop a weighted genetic risk score (GRS). Functionality of the susceptibility genetic variants were investigated.

\section{Methods}

\section{Ethics statement}

The study was approved by the Institutional Review Board of the University of Hong Kong/Hospital Authority Hong Kong West Cluster.

\section{Study design}

Data from three study cohorts were used in the present meta-analysis. One cohort was the latest study conducted by the China Consortium for the Genetics of Autoimmune Thyroid Disease (7) (Shanghai Cohort). While another published cohort was our previous GWAS with study participants recruited from the Southern
Chinese population in Hong Kong (8) (HK Cohort A), a new dataset, namely HK Cohort B, was also recruited in Hong Kong using the same selection criteria. Participants in all the study cohorts gave informed consent and ethics approval was obtained from the respective institutional review board. Details of individual cohorts were described elsewhere and in Supplementary Methods 1 (see section on supplementary material given at the end of the article) (https://osf.io/y9pvr/ (9)). In brief, individuals with TPP presented with thyrotoxicosis and paralysis were recruited as cases while healthy subjects were recruited as controls. The same quality control criteria were applied to the genotype data of the three individual cohorts (Supplementary Methods 2, https://osf.io/y9pvr/ (9)). They were uniformly imputed with reference to Haplotype Reference Consortium (HRC) reference panel (Supplementary Methods 3, https://osf.io/y9pvr/ (9)). After conducting logistic regression analysis in each cohort, the data was combined by fixed-effect meta-analyses (Supplementary Methods 4, https://osf.io/y9pvr/ (9)).

\section{Joint and conditional association analysis}

To identify independent TPP-associated variants reaching the genome-wide significance threshold of $P<5 \times 10^{-8}$, GCTA-COJO (10) was applied to perform a genome-wide stepwise selection procedure in the meta-analysis data, with correction of linkage disequilibrium (LD) structure derived from the genotypes of the cohorts. The joint effects of all selected SNPs were estimated after the model has been optimized. Conditional analysis was performed to assess the independence of association signals within TPP loci. Conditional on the independent TPP-associated variants identified from the stepwise selection procedure, single-SNP association analysis was conducted per risk locus. Restricted maximum likelihood (REML) method was employed to compute the phenotypic variance explained by the independent SNPs (11).

\section{Prediction of TPP risk based on the genotypes of independent SNPs}

With the genotypes of the genome-wide independent TPP-associated SNPs, weighted GRS were computed for all individuals in the cohorts. The GRS were weighted based on the beta estimates derived from HK Cohort A, and they were calculated using the allelic scoring command (--score) in PLINK1.9 (12). Accuracy of TPP prediction was validated in independent cohorts (HK Cohort B and Shanghai Cohort) and evaluated by the area under the receiver 
operating characteristic curves (AUC). The optimal cutoff value for TPP risk classification was determined by the Youden's index, corresponding to a point on the receiver operating curve (ROC) with the highest vertical distance from the diagonal line of the ROC, which intends to maximize overall correct classification rates and minimize misclassification rates. The positive predictive value (PPV) and negative predictive value (NPV) of the prediction models were evaluated for each cohort based on the sensitivity, specificity, as well as the prevalence of TPP.

\section{Functional annotation of TPP-associated SNPs}

An integrative web-based tool, Functional Mapping and Annotation (FUMA) (13) version 1.3.6, was employed to annotate the TPP-associated SNPs. In FUMA, combined annotation-dependent depletion (CADD) score (14), RegulomeDB score (15) and ChromHMM-derived chromatin state (16) were computed for the candidate SNPs in LD with the independent SNPs $\left(\mathrm{r}^{2} \geq 0.1\right)$, which are also included in 1000 Genome. SNPs with CADD score greater than the threshold of 12.37 are considered as deleterious (17). SNPs having low RegulomeDB scores imply more likely regulatory roles: score of 1 indicates the SNPs are likely to affect binding and linked to the expression of a gene target; score of 2 indicates the SNPs are likely to affect binding (15). Whereas, chromatin states of 1-7 derived by ChromHMM imply the openness of chromatin and thus higher accessibility (16). To prioritize SNPs for further functional analysis, candidate SNPs meeting at least two out of the three aforementioned criteria ((i) CADD score $\geq 12.37$, (ii) RegulomeDB score $\leq 2$; (iii) chromatin states $1-7)$ are further investigated. In case none of the candidate SNPs within the same risk locus met the above criteria, the independent SNP itself would be investigated. Further functional investigations included expression quantitative trait loci (eQTL) analysis by current release (V8) of Genotype-Tissue Expression (GTEx) portal (18). Significant eQTL was defined as those having a false discovery rate $(\mathrm{FDR})<0.05$. Three-dimensional DNADNA interactions between genomic region containing the candidate SNPs and genes (enhancer or promoter of genes, which may be outside the disease risk loci) were also examined using Hi-C data from a comprehensive survey of chromatin organization in human tissues conducted by the Roadmap Epigenomics Consortium (GSE87112 in Gene Expression Omnibus) (19). Only interactions with FDR $<1 \times 10^{-6}$ were considered as significant. With the summary statistics from GWAS meta-analysis, FUMA also performed a gene-set enrichment analysis using the built-in MAGMA tool (20), providing insights regarding the pathways or cellular functions involved in the genetic etiology of TPP. In MAGMA v1.07, individual genes were aggregated to 15496 sets of genes with shared biological, functional, or other characteristics (from MsigDB v7.0, including 5500 curated gene sets/canonical pathways and 9996 Gene Ontology terms (biological processes, cellular components and molecular functions)). A gene-set is considered significantly enriched if it can pass the threshold of multiple-testing $(P<0.05 / 15$ $\left.496=3.227 \times 10^{-6}\right)$.

\section{Results}

\section{Meta-analysis of GWAS}

Imputation and quality control criteria (Supplementary Table 1, https://osf.io/y9pvr/ (9)) were applied to the three individual cohorts. To account for population stratification, HK Cohort A, HK Cohort B and Shanghai Cohort were adjusted for the first one, three and one principal components, respectively. A total of 3835 individuals (319 cases and 3516 controls) and 7077246 SNPs were included in the genome-wide meta-analysis. Among the cases, 306 individuals (95.92\%) are male (Table 1). The QQ plot (Fig. 1A) demonstrated a substantial number of SNPs were observed to have $P$-values lower than expected under the null hypothesis. The genomic inflation factor $(\lambda)$ was 1.012, suggesting unmodeled population structure was unlikely. There were 260 SNPs which reached genome-wide significance $\left(P<5 \times 10^{-8}\right)$ in the meta-analysis (Supplementary Table 2, https:/osf.io/ y9pvr/ (9)), represented by four independent SNPs (Table 2) in four distinct genomic loci at 4q31.3, 6p21.33-p21.22, 16q22.3 and 17q24.3 (Supplementary Table 3, https:// osf.io/y9pvr/ (9)) as shown in the Manhattan Plot (Fig. 1B). These SNPs could no longer reach the genomewide significance level when the association analysis was conditioned on the independent SNP on the same chromosome (Supplementary Table 2, https://osf.io/ y9pvr/ (9)), indicating that each of the four genetic loci represented a single independent signal for TPP. The four independent SNPs explained 5.69\% of the genetic liability of TPP in REML analysis.

A joint association analysis was performed for the four independent TPP-associated SNPs (Table 2). The SNP with the strongest association signal was rs312743 (C-allele: $\mathrm{OR}=2.564 ; 95 \%$ CI: $2.107-3.119 ; P=4.82 \times 10^{-21}$ ) near KCNJ2 at chromosomal region $17 \mathrm{q} 24.3$, which was also reported by us and others $(5,6,7,8)$. Although 


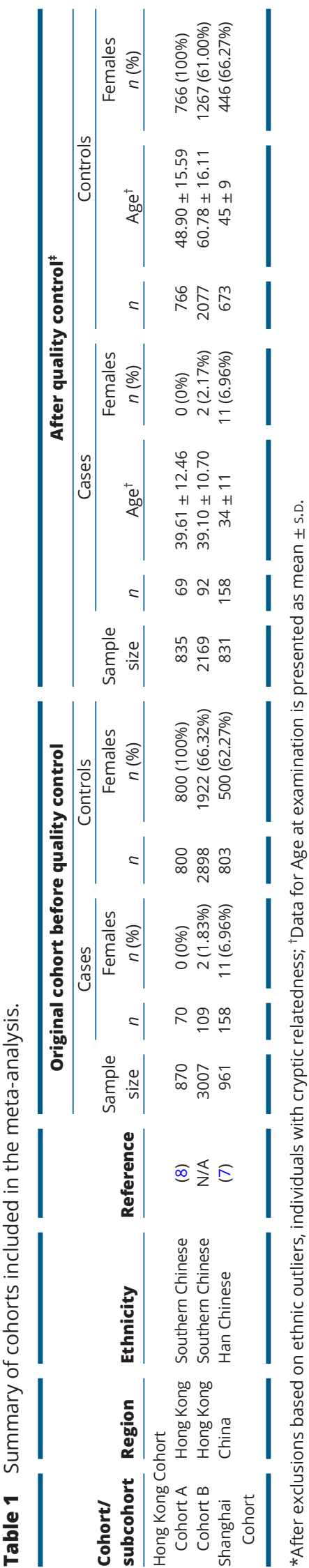

rs112723370 was associated with TPP risk (T-allele: $\left.\mathrm{OR}=2.558 ; 95 \% \mathrm{CI}: 1.897-3.482 ; P=1.11 \times 10^{-9}\right)$, it was downstream of HLA Complex P5 (HCP5) at 6p21.33p21.32 and within the major histocompatibility complex $(\mathrm{MHC})$. Both HCP5 and MHC are known locus for GD (21, 22). Thus, it might represent a risk locus for GD instead of TPP and was excluded from subsequent functional annotation and TPP prediction model. The remaining two independent SNPs were located at novel susceptibility loci. The rs6827197 SNP (T-allele: OR $=4.074 ; 95 \% \mathrm{CI}$ : $\left.2.553-6.504 ; P=3.90 \times 10^{-9}\right)$ at $4 \mathrm{q} 31.3$ was $37 \mathrm{~kb}$ upstream of the gene tripartite motif-containing 2 (TRIM2). The rs6420387 SNP (T-allele: OR $=1.861 ; 95 \%$ CI: $1.494-2.319$; $P=2.97 \times 10^{-8}$ ) was near $A C 140912.1$ at $16 \mathrm{q} 22.3$. After excluding rs112723370 at the GD locus, the remaining three independent SNPs accounted for $4.36 \%$ of the genetic liability of TPP. Regional association plots of SNPs within $250 \mathrm{~kb}$ centering on the independent SNPs are shown in Fig. 2.

\section{Meta-analysis comprising male cases and all healthy controls}

Due to the higher prevalence of TPP in male, metaanalysis was also conducted in male cases and all healthy controls. A total of 3822 individuals (306 male TPP cases and 3516 healthy controls) and 7076963 SNPs were included in this meta-analysis. The QQ and Manhattan plots were generated (Supplementary Fig. 1, https://osf. io/y9pvr/ (9)), with $\lambda$ of 1.016. There were 369 genomewide significant SNPs (Supplementary Table 4, https:// osf.io/y9pvr/ (9)), represented by three independent SNPs (Supplementary Table 5, https://osf.io/y9pvr/ (9)) at 4q31.3, 6p21.33 and 17q24.3. Moreover, two loci at 10q22.3 (downstream of C10orf11 or LeucineRich Melanocyte Differentiation Associated (LRMDA)) and 16q22.3 almost reached genome-wide significance (T-allele of rs144097453 at 10q22.3: OR $=4.233 ; 95 \%$ CI: $2.499-7.170 ; P=8.08 \times 10^{-8}$; T-allele of rs6420387 at 16q22.3: OR=1.822; 95\% CI: $1.462-2.271 ; P=9.23 \times 10^{-8}$; Supplementary Table 6, https://osf.io/y9pvr/ (9)).

\section{Functional annotation}

Functions of the genetic variants were annotated using FUMA (Supplementary Table 7, https://osf.io/y9pvr/ (9)). Out of the 469 SNPs available in 1000 Genome and in LD with the three independent SNPs at 4q31.3, 16q22.3 or $17 q 24.3$, the majority of them (99.15\%) were intergenic or intronic (Supplementary Fig. 2A, https://osf.io/y9pvr/ (9)). 
A

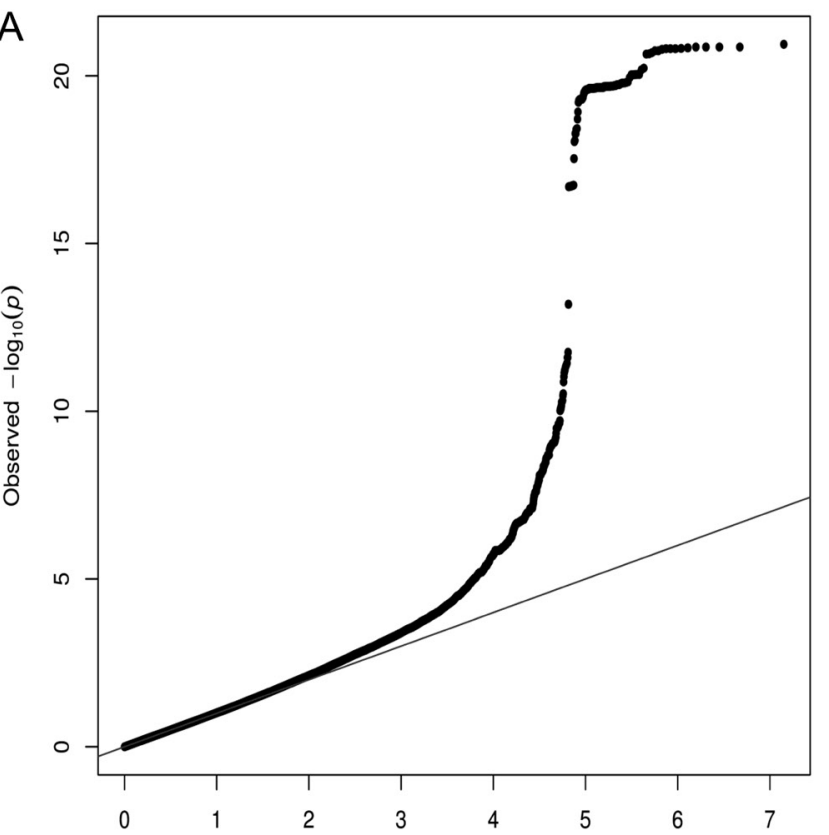

B

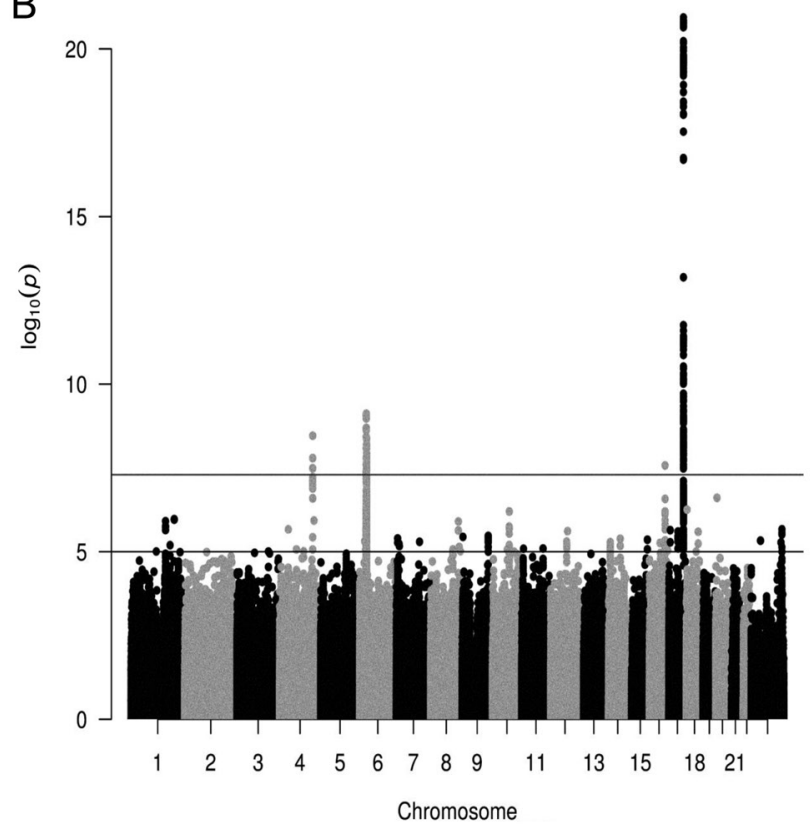

Figure 1

Quantile-quantile (QQ) plot and Manhattan plot of metaanalysis of genome-wide association studies of TPP. (A) The QQ plot showed the deviation of the observed $P$-values from the null hypothesis, with the diagonal representing the expected distribution of $P$-values under the null hypothesis. (B) The Manhattan plot displayed the association of each SNP, with their genomic position on the $\mathrm{x}$-axis and strength of association on the $y$-axis. The upper and lower horizontal lines represent the threshold of genome-wide $\left(P=5 \times 10^{-8}\right)$ and suggestive $\left(P=1 \times 10^{-5}\right)$ significance, respectively.
Fifteen of the candidate SNPs (3.2\%) obtained a CADD score greater than the deleteriousness threshold of 12.37 (17) (Supplementary Fig. 2B, https://osf.io/y9pvr/ (9)). Although a relatively small portion of the SNPs have a low RegulomeDB score (which imply a more likely regulatory role) (Supplementary Fig. 2C, https://osf.io/y9pvr/ (9)), $74.41 \%$ of the SNPs had a chromatin state of 1-7, implying the openness of chromatin and thus higher accessibility (Supplementary Fig. 2D, https://osf.io/y9pvr/ (9)). Two and eleven SNPs at 16q22.3 and 17q24.3, respectively, met two out of the three criteria of having a potential functional role. Together with the independent SNP rs6827197 at 4q31.3, eQTL analyses were performed for a total of 14 SNPs and the significant QTL with FDR $<0.05$ were identified (Supplementary Table 8, https://osf.io/ y9pvr/ (9)). The TPP-risk increasing alleles of candidate SNPs at 4q31.3 and 17q24.3 were associated with increased and reduced expression of TRIM 2 and KCNJ2 in nerve and skeletal muscle respectively. There were 1702 reported pairs of DNA-DNA interactions between genomic region containing the candidate SNPs and 97 nearby/distant genes (including KCNJ2) in various tissues (such as neural progenitor cells, hippocampus and dorsolateral prefrontal cortex) (Supplementary Table 9, https://osf.io/y9pvr/ (9)). MAGMA showed that the top two enriched gene-sets were related to plasma lipoprotein remodeling, and assembly of active lipoprotein lipase and hepatic triacylglycerol lipase complexes. However, they could not pass the threshold of multiple testing in the enrichment analysis (Supplementary Table 10, https://osf.io/y9pvr/ (9)).

\section{Prediction of TPP}

The three genome-wide independent SNPs identified from the whole study population were used to develop a GRS weighted on the beta estimates derived from HK Cohort A. Performance of the prediction model was evaluated (Fig. 3A and B). The AUC in the original HK Cohort A was 0.827. In HK Cohort $A$, the Youden index identified the optimal cut-off at 0.119 , with a sensitivity, specificity, PPV and NPV of $71.014 \%, 77.415 \%, 32.966 \%$ and $90.573 \%$, respectively. To avoid overfitting, we evaluated the accuracy of TPP prediction in two independent cohorts. In general, AUCs in HK Cohort B (0.682) and Shanghai Cohort (0.643) were not as high as that of HK Cohort A (Fig. 3A and B). As candidate SNPs at 4q31.3 and 17 q24.3 were associated with altered gene expression, an additional weighted GRS was computed using rs6827197 and rs312743 only. The resulting prediction model had a generally lower AUC (HK Cohort A: 0.815; HK Cohort B: 

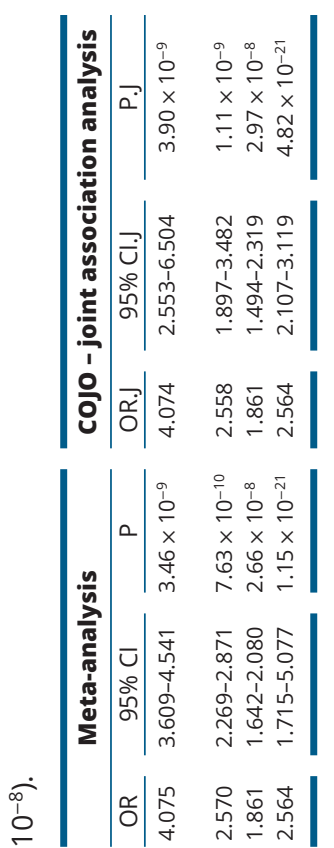

ก แ แ กิ กัฒกิ

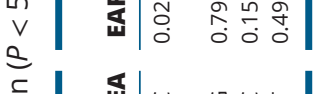

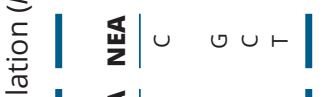

$\frac{\pi}{5}|\mathbb{\Xi}| \vdash \vdash \vdash \cup \mid$

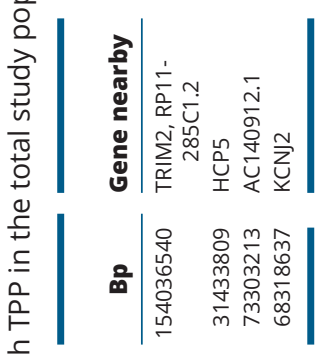

善|

ष्ष

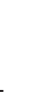

\section{Discussion}

To our knowledge, this GWAS meta-analysis of TPP comprising 319 cases and 3516 controls is the largest GWAS of TPP to-date. Out of the three susceptibility loci identified, two loci near TRIM2 at 4q31.3 and AC140912.1 at 16q22.3 were novel. We also replicated the TPP susceptibility genetic variants near KCNJ2 at 17q24.3. A weighted GRS using the independent SNPs was developed to predict the risk of TPP. The AUC in the original HK Cohort A was $>0.8$, while it was validated in HK Cohort $B$ and the Shanghai Cohort with AUC of 0.682 and 0.643 respectively. Functional analyses suggested that genetic variants at the risk loci might contribute to disease pathogenesis by increasing and reducing expression of TRIM2 in nerve and KCNJ2 in skeletal muscles, respectively.

The genome-wide independent SNP rs6827197 at $4 \mathrm{q} 31.3$ was intergenic and it was around $37 \mathrm{~kb}$ upstream of TRIM2. Approximately $1.2 \mathrm{Mb}$ upstream of the previously reported locus of rs1352714 at DCHS2 (7), these two SNPs were independent $\left(r^{2}=0.003\right)$, suggesting that $r 6827197$ near TRIM2 was a novel risk locus of TPP. In the present study, the risk-increasing T-allele had a relatively low allele frequency (0.025). The SNP had a RegulomeDB score of 5 and achromatin state of 2 , indicating its potential regulatory role (Supplementary Table 7, https:// osf.io/y9pvr/ (9)). Based on eQTL analysis (GTEx/v8), the T-allele was significantly associated with the increased expression of TRIM2 in nerve $\left(\mathrm{FDR}=8.28 \times 10^{-36}\right)$, while TRIM2 was broadly expressed in brain and thyroid. Clinically, mutations in TRIM2 have been implicated in bilateral vocal cord paralysis (23), early onset of CharcotMarie-Tooth disease (CMT) (23) and childhood-onset axonal neuropathy (24). Meanwhile, a SNP in TRIM2 was significantly associated with multiple sclerosis (25) characterized by weakness in limbs, tremor, lack of coordination or unsteady gait. Mice deficient in TRIM2 protein experienced intention tremor, followed by gait ataxia, and episodes of generalized seizures (26). Overexpression of TRIM2 in cultured hippocampal neurons of mouse embryos induced neuron hyperpolarization (27). These studies indicate a strong functional relevance of TRIM2 in TPP pathogenesis. Moreover, TRIM2 was reported to be a target gene of the bioactive form of vitamin $\mathrm{D}(1,25(\mathrm{OH}) 2 \mathrm{D} 3)$, and TRIM2 has a vitamin $\mathrm{D}$ response element in its promoter region (28). Vitamin D 
A

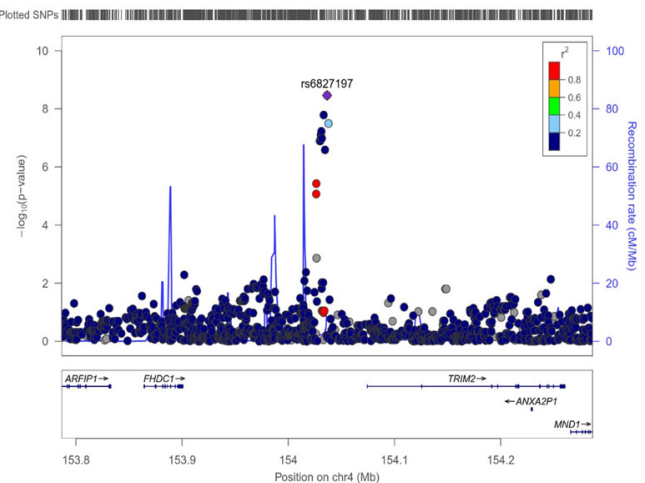

B
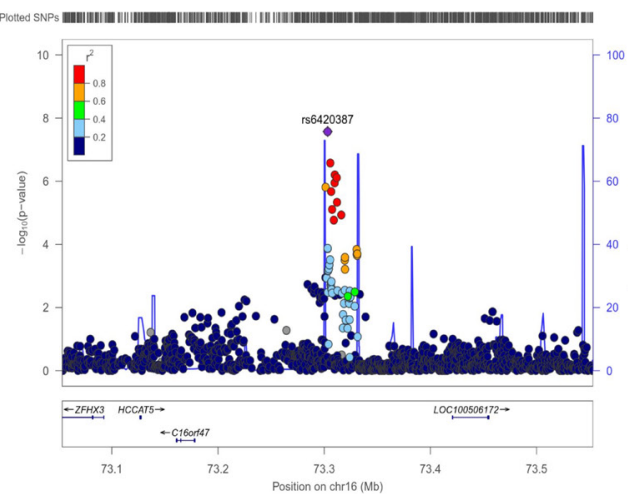

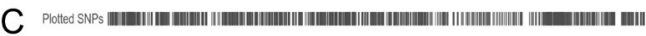
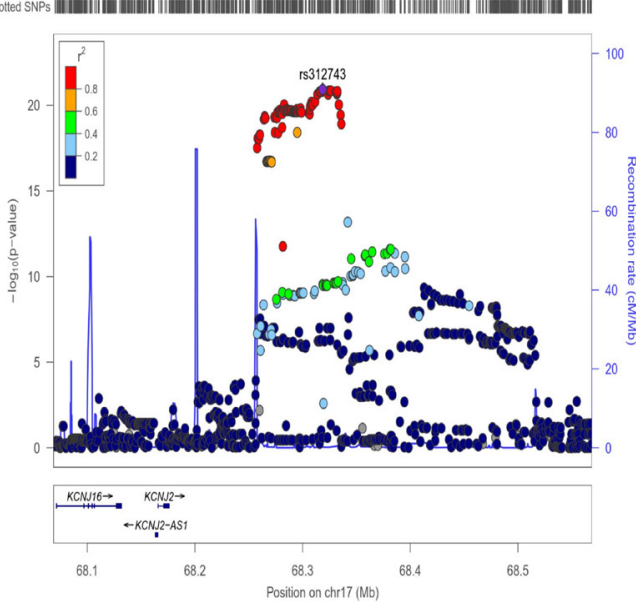

\section{Figure 2}

Regional association plots for the TPP risk loci. The left $y$-axis indicates-log $P$-value of each SNP within $250 \mathrm{~kb}$ upstream/ downstream of the independent SNP. The right $y$-axis indicates the recombination rates estimated from the 1000 genome project (hg19). The annotated genes are indicated at the bottom of the figure. (A) Regional association plot for Chr4q31.3 centering on the independent SNP, rs6827197 (purple diamond). (B) Regional association plot for Chr16q22.3 centering on the independent SNP, rs6420387 (purple diamond). (C) Regional association plot for Chr17q24.3 centering on the independent SNP, rs312743 (purple diamond).

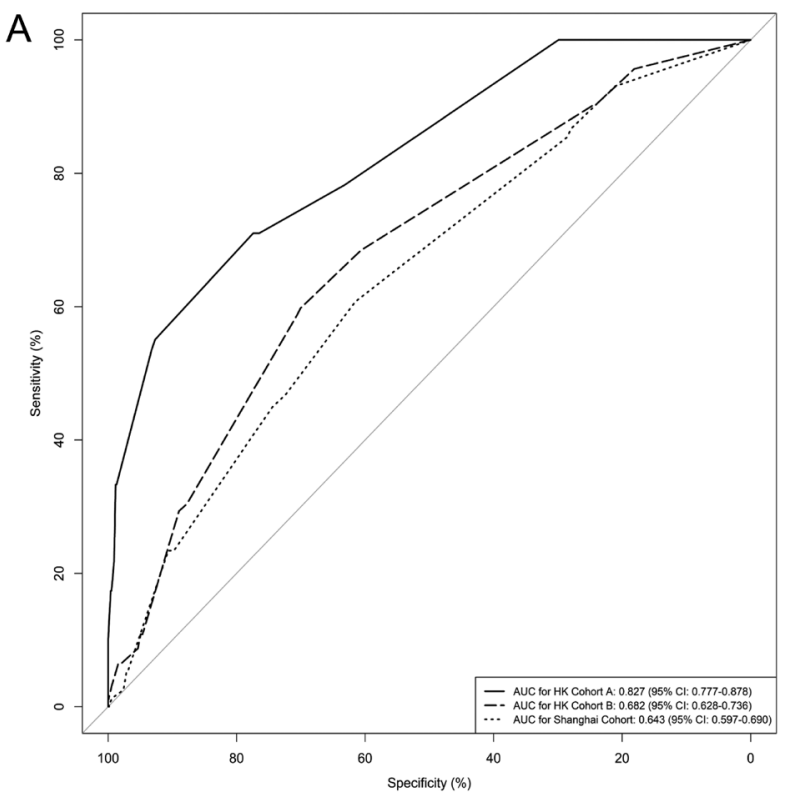

B
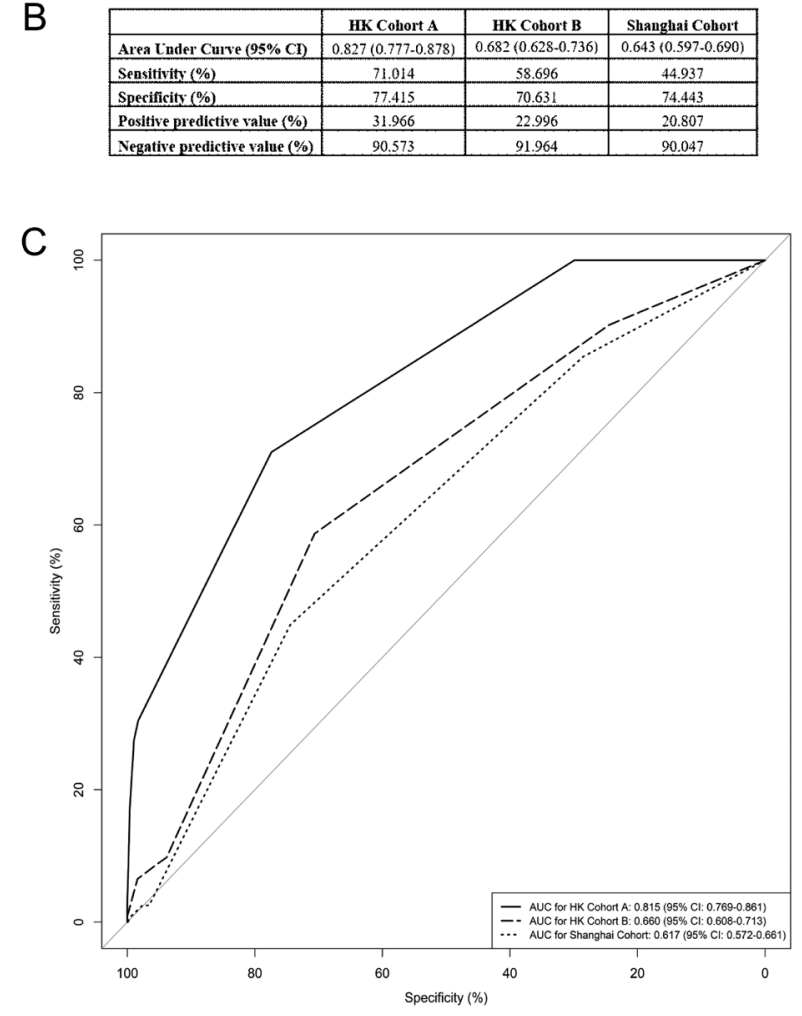

Figure 3

Performance of TPP prediction in the three cohorts by weighted genetic risk score. (A) Comparison of AUC in the three cohorts by weighted genetic risk score. (B) Performance metrics of prediction model in the three cohorts. (C) Comparison of AUC in the three cohorts by weighted genetic risk score with two independent SNPs that altered expression of TRIM2 and KCNJ2. 
metabolism was shown to be over-represented (ranked thirdamong all gene-sets; $P=1.58 \times 10^{-4}$; Supplementary Table 10, https://osf.io/y9pvr/ (9)) in the gene-set enrichment analysis, although it could not pass the multiple testing criteria. Given that vitamin D deficiency is known to be associated with Graves' disease (29) and it may contribute to muscle weakness (30), further studies are warranted to examine if vitamin D metabolism and TRIM2 regulation were involved in the pathogenesis of TPP.

The candidate gene AC140912.1 at 16q22.3 was broadly expressed in brain, with the highest expression in basal ganglia (GTEx/v8). The risk-increasing T-allele of rs6420387 is significantly associated with increased expression of AC140912.1 in basal ganglia $(P=0.02)$, which may be involved in voluntary control of body movement. The candidate gene LRMDA at 10q22.3 that approached genome-wide significance in the metaanalysis of male cases and all controls had ubiquitous expression in adrenal gland, thyroid and spleen (GTEx/ v8). Nonsense mutation in the gene was associated with nystagmus (31), a visual condition when the eyes make involuntary, uncontrolled and repetitive movement. Yet, further investigation on the relationship between TPP and these candidate genes are warranted.

TPP is widely recognized as a channelopathy. This is well-supported by the identified susceptibility/candidate genes of TPP in human, such as KCNJ2 $(5,6,7,8)$, KCNJ18 (4), voltage-dependent calcium channel $\left(\mathrm{Ca}_{\mathrm{v}} 1.1\right)(32)$, and voltage-gated sodium channel $\left(\mathrm{Na}_{\mathrm{v}} 1.4\right)$ (33). Although a study identified a susceptibility variant located in ncRNA CTD-2378E21.1, it is downstream of KCNJ2 (6). To the best of our knowledge, the only reported genome-wide significant TPP-associated variant that was unrelated to ion channel genes was a variant in DCHS2 identified in a single Cohort, without much functional relevance with TPP (7). However, the locus did not reach genome-wide significance in the current meta-analysis. Taken together, our study has identified two novel and robust genomewide significant TPP loci near TRIM2 and AC140912.1 which are unrelated to ion channels, providing evidence that TPP may not be solely a channelopathy.

Notably, among the TPP susceptibility genes, mutations near TRIM2 and KCNJ2 were implicated in monogenic disorders, namely CMT and ATS, respectively. Our study provides an insight that common variants in proximity of genes implicated in monogenic muscular paralysis disorders, such as CMT and ATS, might dysregulate the expression of these genes, leading to milder phenotypes observed in TPP. In addition, environmental exposures, such as strenuous exercise, intake of carbohydrate-rich meals or alcohol, are precipitating factors of TPP attack (1). Since the three genome-wide independent TPPassociated SNPs accounted for only $4.36 \%$ of genetic liability, the gene-environment interaction might explain a large portion of the 'missing heritability' of TPP.

As early detection of TPP might prevent lifethreatening complications, a weighted GRS was developed based on the three genome-wide independent SNPs identified. A prediction model with AUC $\geq 0.7$ was considered clinically useful (34). The weighted GRS in the original HK Cohort A had an AUC of 0.827 , indicating that this GRS is potentially clinically useful. However, as the model was evaluated in the cohort from which the model is developed, there was an overfitting problem, leading to a higher AUC. We, therefore, evaluated the accuracy of TPP prediction in two independent cohorts. AUCs of HK Cohort B and Shanghai Cohort were not as high as that of HK Cohort A. Nevertheless, the post-test probability of TPP was improved (PPVs $\geq 20.807 \%$ and NPV $\geq 90.047 \%$ ), given that the pre-test probability of having TPP and not having TPP is 13 and $87 \%$, respectively (based on the prevalence of $13 \%$ among male patients with thyrotoxicosis in Chinese (1)). Notably, the GRS was developed and validated in cohorts with TPP cases and healthy controls where the prevalence of TPP was lower than that among individuals with thyrotoxicosis. It is expected that the prediction accuracy was underestimated in the cohorts under investigation. Improvement in the accuracy is anticipated if the prediction is conducted among individuals with thyrotoxicosis. On the other hand, the AUC, PPV and NPV of the Shanghai Cohort were the lowest among the tested cohorts, which might be explained by the slight heterogeneity of the genetic architecture between the northern and southern Chinese population in Shanghai and Hong Kong (35). An additional GRS was developed based on the two independent SNPs at $4 \mathrm{q} 31.3$ and $17 \mathrm{q} 24.3$ which altered the expression of TRIM2 and KCNJ2 (rs6827197 and rs312743). Only slightly lower AUC was observed for all the three cohorts in this prediction model, implying that the accuracy of TPP risk prediction was mainly contributed by the two SNPs located within the risk loci implicated in changes of gene expression.

This study has several strengths. It was the largest GWAS meta-analysis to-date, with TPP cases more than double of the latest GWAS (7), leading to the increase in power. Moreover, individual GWAS were imputed again with reference to the HRC panel such that a more comprehensive set of genetic variants were tested, 
enabling the discovery of new risk loci and refining of causal loci (36). One limitation of the present study was that the controls in our study were healthy individuals, while TPP patients also had GD. It may be possible that the risk loci identified were susceptible to GD instead of TPP. Nevertheless, by checking against the published GWAS of GD in different populations, including Han Chinese (21, 37), Japanese (7) and Europeans (38), we confirmed that the TPP risk loci identified in the present study were not GD susceptibility loci. Furthermore, the relatively low prevalence of TPP resulted in difficulties in the recruitment of cases. The relatively small number of cases in the study may lead to false-positive findings. Moreover, the GWAS meta-analysis included subjects of Chinese ethnicity only, implying that the identified susceptibility loci for TPP may be ethnic-specific. Due to the difference in LD patterns, association studies conducted in other ethnicities may not be able to retrieve the same susceptibility variants as identified in the current study. Generalization of the current findings to other ethnicities should be cautious. Replication of the present findings in independent cohorts would be required when such cohorts become available. In addition, due to the higher prevalence of TPP in male and the limited number of healthy male participants available in the cohorts, there was a skewed distribution of gender in the current meta-analysis: more male as cases and more female as controls, respectively. Inclusion of female samples in the controls would underestimate instead of overestimating the association, leading to false-negative rather than false-positive findings. Genderspecific GWAS meta-analysis might explain how genetics might contribute to the higher prevalence of TPP in male. However, with HK Cohort B and Shanghai Cohort, a malespecific GWAS meta-analysis of TPP using only 237 cases and 1037 controls of male participants was not desirable due to the relatively small sample size and low power. The power to detect an association with $\mathrm{OR} \leq 1.5$ is less than 0.07 if the risk allele frequency is within a wide range from 0.01 to 0.95 , based on the calculation using QUANTO (39) (Supplementary Table 11, https://osf.io/y9pvr/ (9)).

In conclusion, the present study reports the first GWAS meta-analysis of TPP with the largest sample size to-date, identifies novel genome-wide significant TPP susceptibility genes at 4q31.3 and 16q22.3 which are unrelated to ion channel. The TPP susceptibility locus near TRIM2 at 4 q31.3 might play a role in the disease pathogenesis by altering the expression of TRIM2 in nerve. Whereas, the function of the locus at $16 \mathrm{q} 22.3$ is yet to be determined. In addition to the previously reported variant near KCNJ2, this meta-analysis identified common variants which altered the expression of genes implicated in monogenic disorder characterized by muscle paralysis, resulting in the milder phenotype of TPP. The weighted GRS had an area under curve of 0.827 and 0.682 in the derivation and validation cohorts in Hong Kong. Including larger sample in future studies are warranted to further refine the causal risk loci.

\section{Supplementary materials}

This is linked to the online version of the paper at https://doi.org/10.1530/ EJE-20-0523.

\section{Declaration of interest}

The authors declare that there is no conflict of interest that could be perceived as prejudicing the impartiality of this study.

\section{Funding}

Supported by National Natural Science Foundation of China (NSFC) (project number: 81661168016), and joint research scheme of NSFC and Research Grants Council (RGC) (project number: N_HKU729/16).

\section{Author contribution statement}

C L C and S H D were involved in study concept and design. G H L and C L C were involved in statistical and bioinformatics analysis of data. $G H L$ and $C$ $L C$ contributed to the drafting of the manuscript. G H L, C L C, Z S X, S H D and $\mathrm{A} W \mathrm{~K}$ were involved in the interpretation of data and critical revision of the manuscript for important intellectual content. C L C contributed to study supervision.

\section{References}

1 Kung AW. Clinical review: thyrotoxic periodic paralysis: a diagnostic challenge. Journal of Clinical Endocrinology and Metabolism 200691 2490-2495. (https://doi.org/10.1210/jc.20060356)

2 Ko GT, Chow CC, Yeung VT, Chan HH, Li JK \& Cockram CS. Thyrotoxic periodic paralysis in a Chinese population. QJM 199689 463-468. (https://doi.org/10.1093/qjmed/89.6.463)

3 Falhammar H, Thoren M \& Calissendorff J. Thyrotoxic periodic paralysis: clinical and molecular aspects. Endocrine 201343 274-284. (https://doi.org/10.1007/s12020-012-9777-x)

4 Ryan DP, da Silva MR, Soong TW, Fontaine B, Donaldson MR, Kung AW, Jongjaroenprasert W, Liang MC, Khoo DH, Cheah JS et al. Mutations in potassium channel Kir2.6 cause susceptibility to thyrotoxic hypokalemic periodic paralysis. Cell 2010140 88-98. (https://doi.org/10.1016/j.cell.2009.12.024)

5 Jongjaroenprasert W, Phusantisampan T, Mahasirimongkol S, Mushiroda T, Hirankarn N, Snabboon T, Chanprasertyotin S, Tantiwong P, Soonthornpun S, Rattanapichart P et al. A genome-wide association study identifies novel susceptibility genetic variation for thyrotoxic hypokalemic periodic paralysis. Journal of Human Genetics 201257 301-304. (https://doi.org/10.1038/jhg.2012.20)

6 Song IW, Sung CC, Chen CH, Cheng CJ, Yang SS, Chou YC, Yang JH, Chen YT, Wu JY \& Lin SH. Novel susceptibility gene for nonfamilial hypokalemic periodic paralysis. Neurology 201686 1190-1198. (https://doi.org/10.1212/WNL.0000000000002524) 
7 Zhao SX, Liu W, Liang J, Gao GQ, Zhang XM, Yao Y, Wang HN, Yuan FF, Xue LQ, Ma YR et al. Assessment of molecular subtypes in thyrotoxic periodic paralysis and Graves disease among Chinese Han adults: a population-based genome-wide association study. JAMA Network Open 20192 e193348. (https://doi.org/10.1001/ jamanetworkopen.2019.3348)

8 Cheung CL, Lau KS, Ho AY, Lee KK, Tiu SC, Lau EY, Leung J, Tsang MW, Chan KW, Yeung CY et al. Genome-wide association study identifies a susceptibility locus for thyrotoxic periodic paralysis at 17q24.3. Nature Genetics 201244 1026-1029. (https://doi. org/10.1038/ng.2367)

9 Li GH \& Cheung CL. Genome-wide meta-analysis of thyrotoxic periodic paralysis - Supplementary Data: OSF [updated 13/07/2020]; 2020. (available at: https://osf.io/y9pvr/)

10 Yang J, Ferreira T, Morris AP, Medland SE, Genetic Investigation of ANthropometric Traits (GIANT) Consortium; DIAbetes Genetics Replication And Meta-analysis (DIAGRAM) Consortium, Madden PA, Heath AC, Martin NG et al. Conditional and joint multiple-SNP analysis of GWAS summary statistics identifies additional variants influencing complex traits. Nature Genetics 201244 369-375, S1-3. (https://doi.org/10.1038/ng.2213)

11 Yang J, Benyamin B, McEvoy BP, Gordon S, Henders AK, Nyholt DR, Madden PA, Heath AC, Martin NG, Montgomery GW et al. Common SNPs explain a large proportion of the heritability for human height. Nature Genetics 201042 565-569. (https://doi. org/10.1038/ng.608)

12 Chang CC, Chow CC, Tellier LC, Vattikuti S, Purcell SM \& Lee JJ. Second-generation PLINK: rising to the challenge of larger and richer datasets. GigaScience 20154 7. (https://doi.org/10.1186/s13742-0150047-8)

13 Watanabe K, Taskesen E, van Bochoven A \& Posthuma D. Functiona mapping and annotation of genetic associations with FUMA. Nature Communications 20178 1826. (https://doi.org/10.1038/s41467-01701261-5)

14 Rentzsch P, Witten D, Cooper GM, Shendure J \& Kircher M. CADD: predicting the deleteriousness of variants throughout the human genome. Nucleic Acids Research 201947 D886-D894. (https://doi. org/10.1093/nar/gky1016)

15 Boyle AP, Hong EL, Hariharan M, Cheng Y, Schaub MA, Kasowski M, Karczewski KJ, Park J, Hitz BC, Weng S et al. Annotation of functional variation in personal genomes using RegulomeDB. Genome Research 201222 1790-1797. (https://doi.org/10.1101/gr.137323.112)

16 Ernst J \& Kellis M. ChromHMM: automating chromatin-state discovery and characterization. Nature Methods 20129 215-216. (https://doi.org/10.1038/nmeth.1906)

17 Amendola LM, Dorschner MO, Robertson PD, Salama JS, Hart R, Shirts BH, Murray ML, Tokita MJ, Gallego CJ, Kim DS et al. Actionable exomic incidental findings in 6503 participants: challenges of variant classification. Genome Research 201525 305-315. (https://doi.org/10.1101/gr.183483.114)

18 Consortium GT. The genotype-tissue expression (GTEx) project. Nature Genetics 201345 580-585. (https://doi.org/10.1038/ng.2653)

19 Schmitt AD, Hu M, Jung I, Xu Z, Qiu Y, Tan CL, Li Y, Lin S, Lin Y, Barr CL et al. A compendium of chromatin contact maps reveals spatially active regions in the human genome. Cell Reports 201617 2042-2059. (https://doi.org/10.1016/j.celrep.2016.10.061)

20 de Leeuw CA, Mooij JM, Heskes T \& Posthuma D. MAGMA: generalized gene-set analysis of GWAS data. PLoS Computational Biology 201511 e1004219. (https://doi.org/10.1371/journal. pcbi.1004219)

21 Chu X, Pan CM, Zhao SX, Liang J, Gao GQ, Zhang XM, Yuan GY, Li CG, Xue LQ, Shen M et al. A genome-wide association study identifies two new risk loci for Graves' disease. Nature Genetics 2011 43 897-901. (https://doi.org/10.1038/ng.898)

22 Lane LC, Kus A, Bednarczuk T, Bossowski A, Daroszewski J, JureckaLubieniecka B, Cordell HJ, Pearce SHS, Cheetham T \& Mitchell AL.
An intronic HCP5 variant is associated with age of onset and susceptibility to Graves disease in UK and Polish cohorts. Journal of Clinical Endocrinology and Metabolism 2020105 e3277-e3284. (https://doi.org/10.1210/clinem/dgaa347)

23 Pehlivan D, Coban Akdemir Z, Karaca E, Bayram Y, Jhangiani S, Yildiz EP, Muzny D, Uluc K, Gibbs RA, Baylor-Hopkins Center for Mendelian et al. Exome sequencing reveals homozygous TRIM2 mutation in a patient with early onset CMT and bilateral vocal cord paralysis. Human Genetics 2015134 671-673. (https://doi. org/10.1007/s00439-015-1548-3)

24 Ylikallio E, Poyhonen R, Zimon M, De Vriendt E, Hilander T, Paetau A, Jordanova A, Lonnqvist T \& Tyynismaa H. Deficiency of the E3 ubiquitin ligase TRIM2 in early-onset axonal neuropathy. Human Molecular Genetics 201322 2975-2983. (https://doi. org/10.1093/hmg/ddt149)

25 International Multiple Sclerosis Genetics Consortium. Genome-wide association study of severity in multiple sclerosis. Genes and Immunity 201112 615-625. (https://doi.org/10.1038/gene.2011.34)

26 Balastik M, Ferraguti F, Pires-da Silva A, Lee TH, Alvarez-Bolado G, Lu KP \& Gruss P. Deficiency in ubiquitin ligase TRIM2 causes accumulation of neurofilament light chain and neurodegeneration. PNAS 2008105 12016-12021. (https://doi.org/10.1073/ pnas.0802261105)

27 Khazaei MR, Bunk EC, Hillje AL, Jahn HM, Riegler EM, Knoblich JA, Young P \& Schwamborn JC. The E3-ubiquitin ligase TRIM2 regulates neuronal polarization. Journal of Neurochemistry 2011117 29-37. (https://doi.org/10.1111/j.1471-4159.2010.06971.x)

28 Wang TT, Tavera-Mendoza LE, Laperriere D, Libby E, MacLeod NB, Nagai Y, Bourdeau V, Konstorum A, Lallemant B, Zhang R et al. Large-scale in silico and microarray-based identification of direct 1,25-dihydroxyvitamin D3 target genes. Molecular Endocrinology 2005 19 2685-2695. (https://doi.org/10.1210/me.2005-0106)

$29 \mathrm{Kim} \mathrm{D}$. The role of vitamin D in thyroid diseases. International Journal of Molecular Sciences 201718 1949. (https://doi.org/10.3390/ ijms18091949)

30 Dzik KP \& Kaczor JJ. Mechanisms of vitamin D on skeletal muscle function: oxidative stress, energy metabolism and anabolic state. European Journal of Applied Physiology 2019119 825-839. (https://doi. org/10.1007/s00421-019-04104-x) )

31 Gronskov K, Dooley CM, Ostergaard E, Kelsh RN, Hansen L, Levesque MP, Vilhelmsen K, Mollgard K, Stemple DL \& Rosenberg T. Mutations in c10orf11, a melanocyte-differentiation gene, cause autosomal-recessive albinism. American Journal of Human Genetics 201392 415-421. (https://doi.org/10.1016/j. ajhg.2013.01.006)

32 Kung AW, Lau KS, Fong GC \& Chan V. Association of novel single nucleotide polymorphisms in the calcium channel alpha 1 subunit gene $(\mathrm{Ca}(\mathrm{v}) 1.1)$ and thyrotoxic periodic paralysis. Journal of Clinical Endocrinology and Metabolism 200489 1340-1345. (https://doi. org/10.1210/jc.2003-030924)

33 Lane AH, Markarian K \& Braziunene I. Thyrotoxic periodic paralysis associated with a mutation in the sodium channel gene SCN4A. Journal of Pediatric Endocrinology and Metabolism 200417 1679-1682. (https://doi.org/10.1515/jpem.2004.17.12.1679)

34 McDowell I. Measuring Health: A Guide to Rating Scales and Questionnaires. Oxford University Press, 2006.

35 Wang Y, Lu D, Chung YJ \& Xu S. Genetic structure, divergence and admixture of Han Chinese, Japanese and Korean populations. Hereditas 2018155 19. (https://doi.org/10.1186/s41065-0180057-5)

36 McCarthy S, Das S, Kretzschmar W, Delaneau O, Wood AR, Teumer A, Kang HM, Fuchsberger C, Danecek P, Sharp K et al. A reference panel of 64,976 haplotypes for genotype imputation. Nature Genetics 2016 48 1279-1283. (https://doi.org/10.1038/ng.3643)

37 Zhao SX, Xue LQ, Liu W, Gu ZH, Pan CM, Yang SY, Zhan M, Wang HN, Liang J, Gao GQ et al. Robust evidence for five new 
Graves' disease risk loci from a staged genome-wide association analysis. Human Molecular Genetics 201322 3347-3362. (https://doi. org $/ 10.1093 / \mathrm{hmg} / \mathrm{ddt} 183$ )

38 Cooper JD, Simmonds MJ, Walker NM, Burren O, Brand OJ, Guo H, Wallace C, Stevens H, Coleman G, Wellcome Trust Case Control Consortium et al. Seven newly identified loci for autoimmune thyroid disease. Human Molecular Genetics 201221 5202-5208. (https://doi.org/10.1093/hmg/ dds357)

39 Gauderman WJ \& Morrison JM. QUANTO 1.1: a computer program for power and sample size calculations for genetic-epidemiology studies, 2006.

Received 14 May 2020

Revised version received 30 July 2020

Accepted 28 September 2020 